\title{
Two-dimensional sonic crystals with Helmholtz resonators
}

\author{
Xinhua $\mathrm{Hu}^{*}$ and C. T. Chan \\ Department of Physics, Hong Kong University of Science and Technology, Clear Water Bay, Kowloon, Hong Kong, China \\ Jian Zi \\ Surface Physics Laboratory (National Key Lab), Fudan University, Shanghai 200433, People's Republic of China
}

(Received 29 November 2004; revised manuscript received 23 March 2005; published 5 May 2005)

\begin{abstract}
We present a type of sonic crystal composed with an array of two-dimensional Helmholtz resonators, which in the long-wave regime have both a high relative acoustic refractive index $n$ and at the same time, a small acoustic impedance $Z$ mismatch with air for airborne sound. We analyze the $n$ and $Z$ of such sonic crystals by finite-difference time-domain simulations, and by mapping our results to a corresponding electromagnetic (EM) model, and we find that our Helmholtz resonant sonic crystal has a bigger effective magnetic permeability $\mu$ than the conventional rigid-cylinder sonic crystal in its EM counterpart. As a result, a thin convergent lens with very good focusing effect is demonstrated based on our crystal.
\end{abstract}

DOI: 10.1103/PhysRevE.71.055601

PACS number(s): 43.40.+s, 63.20.-e

While many materials in nature can be used for optical lenses, common solids or liquids are not suitable for fabricating acoustic refractive devices for airborne sound because of their acoustic impedance mismatch with air. In recent years, the concept of band-gap materials has extended our ability to manipulate and control the flow of classical waves [1-5]. In addition to the obvious benefits of a band gap, such as the modification of density of states, band-gap materials basically allow us to have designable dispersions. For example, Cervera et al. recently made a significant progress in the problem by using a sonic crystal (SC) as an acoustic lens, which is composed with a periodic array of rigid cylinders [2].

In addition to Bragg-scattering-type mechanisms that can lead to band gaps and strongly modified dispersions, local resonances can also do the job. The locally resonant sonic crystal is an interesting composite material in which sound propagation is strongly modified by acoustic resonances $[3,4]$. Due to the periodicity, the single resonant frequency will extend to a stop band and the advantage of using resonance to create the gap (in contrast to Bragg scattering) is that the frequency of the gap is decoupled from the periodicity. Liu et al. had demonstrated that a resonant sonic crystal with building blocks of rubber-coated lead balls exhibit a low-frequency sonic band gap, and the resonance can provide a maximum impedance mismatch to shield the airborne sound [3].

Despite of these major advances, a good acoustic lens for airborne sound is still a challenge. The local-resonant sonic crystal creates a giant impedance mismatch, and as such, it is good for sound shielding, but not suitable for lensing. The rigid cylinder sonic crystal (RCSC) is a functional material for an acoustic lens, but $n$ and $Z$ are coupled together: high relative acoustic refractive index $n$ (which is desirable) implies high relative acoustic impedance $Z$ (undesirable). Can we have a composite system that has a higher acoustic $n$, but

\footnotetext{
*Electronic address: xhhu@ust.hk
}

at the same time a smaller impedance mismatch with air? In this paper, we present a class of resonant sonic crystals composed with two-dimensional (2D) Helmholtz resonators (split rigid tubes as shown in the inset of Fig. 1) [6-8], which in the long-wave regime have both a high relative acoustic refractive index $n$ and at the same time, a low relative acoustic impedance $Z$ for airborne sound. We analyze the $n$ and $Z$ of such sonic crystals by finite-difference time-domain (FDTD) simulations [5,9], and by mapping our results to a corresponding electromagnetic (EM) model, and we find that our Helmholtz resonant sonic crystal (HRSC) has a bigger effective magnetic permeability $\mu$ than the conventional RCSC in its EM counterpart. As a result, a thin convergent lens with very good focusing effect is demonstrated based on our crystal.

In a $2 \mathrm{D}$ acoustic system, the acoustic wave equations are

$$
\frac{\partial p(\mathbf{r})}{\partial t}=-\frac{1}{\kappa(\mathbf{r})} \nabla \cdot \mathbf{v}(\mathbf{r})
$$

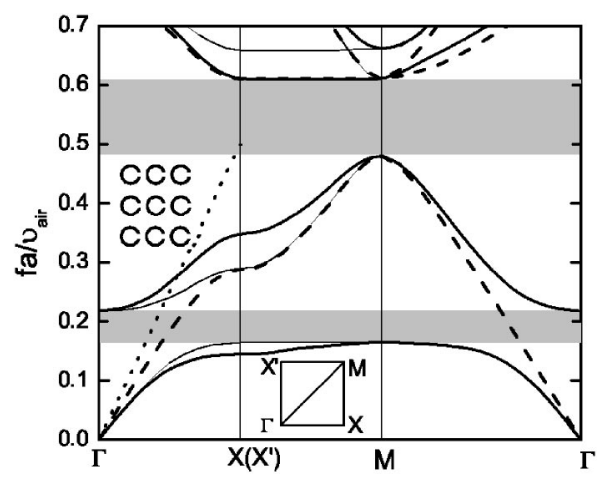

FIG. 1. Acoustic band structures of the HRSC (thick solid lines for $\Gamma-X-M$, thin solid lines for $\Gamma-X^{\prime}-M$ ) and RCSC (dashed lines). The dispersion curve of sound in air along the $\Gamma-X$ direction is plotted as the dotted line. The insets are the structure (left panel) and reduced Brillouin zone (middle panel) of the HRSC. The HRSC has $R=0.4 a, d=\Delta=0.1 a$, and the RCSC has $R=0.4 a$. 


$$
\frac{\partial \mathbf{v}(\mathbf{r})}{\partial t}=-\frac{1}{\rho(\mathbf{r})} \nabla p(\mathbf{r}),
$$

where $\mathbf{r}=(x, y), \kappa$ is compressibility, $\rho$ is density, $p$ is pressure, and $\mathbf{v}$ is the velocity [6]. These equations closely resemble those of TE waves in a $2 \mathrm{D}$ electromagnetic (EM) system

$$
\begin{gathered}
\frac{\partial H_{z}(\mathbf{r})}{\partial t}=-\frac{1}{\mu(\mathbf{r})} \nabla \cdot \mathbf{E}^{\prime}(\mathbf{r}), \\
\frac{\partial \mathbf{E}^{\prime}(\mathbf{r})}{\partial t}=-\frac{1}{\varepsilon(\mathbf{r})} \nabla H_{z}(\mathbf{r}),
\end{gathered}
$$

where $\mu$ is magnetic permeability, $\varepsilon$ is dielectric constant, $H_{z}$ is magnetic field along $z$ axis, $\mathbf{E}^{\prime}=\left(E_{y},-E_{x}\right)$, and $\mathbf{E}$ $=\left(E_{x}, E_{y}\right)$ is the transverse electric field [10]. Thus a 2D acoustic system can be mapped into an EM counterpart, where $p, \mathbf{v}, \rho, \kappa$ correspond to $H_{z}, \mathbf{E}^{\prime}, \varepsilon, \mu$, respectively. Therefore, the well-known FDTD method for EM waves [9] can be directly used to study our 2D air-rigid-body $(\rho \sim \infty)$ acoustic system, which maps exactly to the $2 \mathrm{D}$ vacuumperfect-metal $(\varepsilon \sim \infty)$ EM system.

In the present paper, we consider SCs with a square lattice with a lattice constant of $a$. In Fig. 1 we show the acoustic band structures for a HRSC composed of split tubes with radius $r=0.4 a$, wall thickness $d=0.1 a$, and aperture width $\Delta=0.1 a$, as well as a RCSC made with cylinders with the same radius $r=0.4 a$ for comparison. The band structure is obtained using FDTD calculations by imposing Bloch periodic boundary conditions in a single unit cell, and then apply the Fourier transformation technique [9]. We can see that a common Bragg gap exists with reduced frequencies $f^{\prime}$ $=f a / v_{\text {air }}$ from 0.482 to 0.61 , where $v_{\text {air }}$ is the sound speed in air. This gap will depress the lowest dispersion curve from the dotted line (in air) to the dashed line (for RCSC). The relative effective acoustic refractive index $n$ in the long-wave limit can be determined by $n=v_{\text {air }}|\mathbf{k}| / \omega$, where $\mathbf{k}$ is the Bloch wave vector, which gives $n=1.33$ for the RCSC. However, the aperture in HRSC induces an extra resonance gap from 0.164 to 0.218 . The low band edge for the resonance gap is very flat and has very high density of acoustic modes induced by the Helmholtz resonance (HR) [6,7]. Such a HR gap can obviously serve to further decrease the slope of the first band, leading to a higher $n$ ( $n=1.7$ in our HRSC). We note that the acoustic dispersions will be different along the $\Gamma-X$ and $\Gamma-X^{\prime}$ directions (see Fig. 1) in our HRSC. However, the dispersion remains isotropic in the low-frequency range, as the wave cannot resolve the fine structures in the cylinders in the long-wave limit.

We know that both the relative acoustic refractive index $n$ and the relative acoustic impedance $Z$ are important parameters for acoustic refractive materials $[2,6]$. When a plane wave of sound is incident normally to a homogenous slab with $n$ and $Z$ in air, the pressure transmission coefficient $t$ and the pressure reflection coefficient $r$ are given by [6,11]:

$$
\frac{1}{t}=\cos (n k L)-\frac{i}{2}\left(Z+\frac{1}{Z}\right) \sin (n k L),
$$
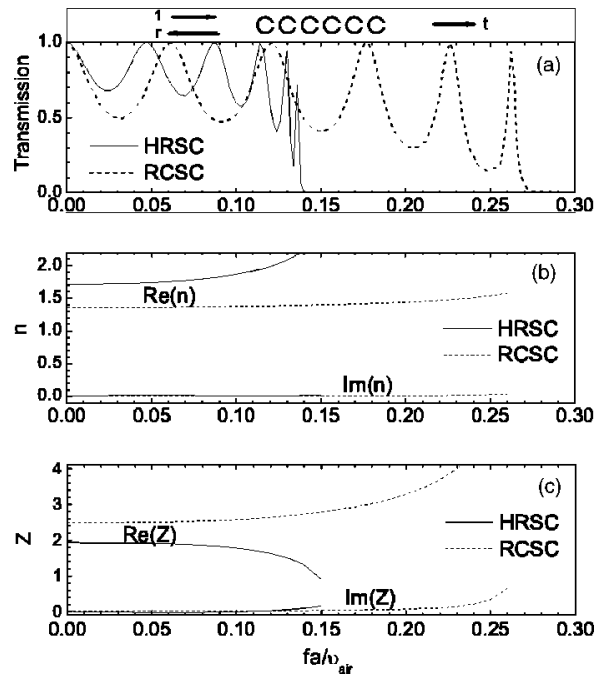

FIG. 2. (a) Transmission spectrum of a slab (six monolayers) made of the HRSC and RCSC with surface normal along the $\Gamma-X$ direction, (b) relative acoustic refractive index $n$, and (c) relative acoustic impedance $Z$ for the first acoustic band of the HRSC and RCSC along the $\Gamma-X$ direction. The SCs are same as those in Fig. 1.

$$
\frac{r}{t}=-\frac{i}{2}\left(Z-\frac{1}{Z}\right) \sin (n k L)
$$

where $k$ is the wave vector of the incident acoustic wave in air and $L$ is the slab thickness. So $n$ and $Z$ can be obtained by $r$ and $t$

$$
\begin{gathered}
Z= \pm \sqrt{\frac{(1+r)^{2}-t^{2}}{(1-r)^{2}-t^{2}}}, \\
n=\frac{1}{k L}\left( \pm \arccos \frac{1-r^{2}+t^{2}}{2 t}+2 \pi m\right) .
\end{gathered}
$$

If the sonic material is passive, $\operatorname{Re}(Z)>0$ and $\operatorname{Im}(n)>0$ will be required, which can determine the sign of $Z$ and $n$. The integer $m$ in Eq. (8) should be determined to yield the same values of $n$ for slabs with different $L$.

We make a transmission calculation for the normal incidence on a slab made of six layers of split tubes or cylinders with the normal in the $\Gamma-X$ direction. In the FDTD calculations, we consider a $15 a \times 1 a$ supercell and use periodic and perfect matched PML absorbing boundary conditions[9] in the upper (bottom) and left (right) borders, respectively (see the inset of Fig. 2). The slab is put in the middle and a monochromatic unidirectional plane-wave source with smooth excitation [9] is incident from the left. Stable $r$ and $t$ can be obtained after 40 periods. In Fig. 2(a) we show the intensity transmission $|t|^{2}$ for the first acoustic band. Due to the Fabry-Perot effect, there are five transmission dips in the spectrum. We can see that our HRSC has higher transmission (68\% at the first dip) than the RCSC (49\% at the first dip). Then we extract the effective $n$ and $Z$ of the SCs from $r$ and $t$ by Eqs. (7) and (8) and plot them in Figs. 2(b) and 2(c) and [11]. Empirically, we found that a layer of cylinders or split tubes is enough to obtain the same results as a thicker SC 
(a)
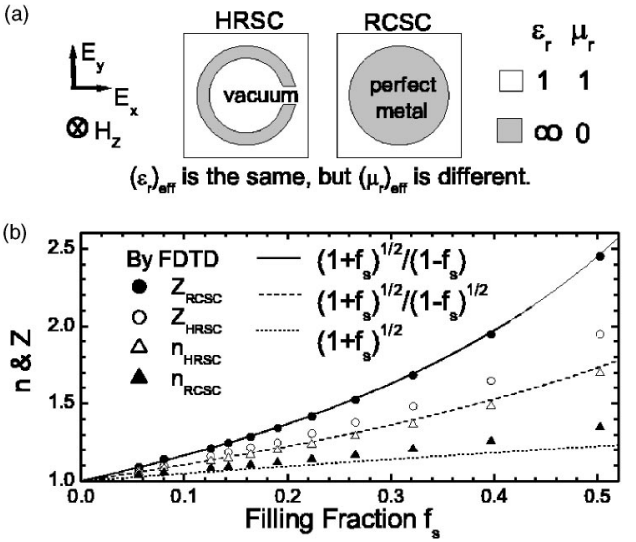

FIG. 3. (a) Analysis of SCs using their EM couterparts. (b) Acoustic refractive index $n$ and acoustic impedance $Z$ of HRSCs and RCSCs in the long-wave regime as a function of filling fraction.

slab. Both $n$ and $Z$ are nearly real except near the band edge, where the multiple scattering effect is strong and an effective medium cannot provide a good description. In the long-wave limit, we can see that our HRSC has a higher $n=1.72$ than the RCSC with $n=1.34$, consistent with the above dispersion calculations. Meanwhile, our HRSC has a smaller $Z=1.95$ than the RCSC with $Z=2.46$, supporting the higher transmission of our HRSC shown in Fig. 2(a).

In order to understand qualitatively the difference in acoustic properties between HRSCs and RCSCs, we map the acoustic systems into their EM counterparts [metal split tube arrays and metal cylinder arrays in vacuum, see Fig. 3(a)], by

$$
\begin{gathered}
n_{a c}=\sqrt{\left(\rho_{r}\right)_{e f f}} \sqrt{\left(\kappa_{r}\right)_{e f f}} \rightarrow \sqrt{\left(\varepsilon_{r}\right)_{e f f}} \sqrt{\left(\mu_{r}\right)_{e f f}}, \\
Z_{a c}=\sqrt{\left(\rho_{r}\right)_{e f f}} \sqrt{\left(\kappa_{r}\right)_{e f f}} \rightarrow \sqrt{\left(\varepsilon_{r}\right)_{e f f}} / \sqrt{\left(\mu_{r}\right)_{e f f}},
\end{gathered}
$$

where the subscript $r$ indicates the relative value compared with air or vacuum (the same below). Note that the definition of acoustic impedance $Z_{a c} \equiv|p| /|\mathbf{v}|$ is different with that for EM impedance $Z_{E M} \equiv\left|\mathbf{E}^{\prime}\right| /\left|H_{z}\right|$ and thus $Z_{a c}$ relates to $1 / Z_{E M}[6,10]$.

In the 2D EM counterpart with TE waves, $\left(\varepsilon_{r}\right)_{\text {eff }}$ and $\left(\mu_{r}\right)_{\text {eff }}$ can be obtained by $[10,12]$

$$
\begin{gathered}
\left(\varepsilon_{r}\right)_{e f f}=\left(D_{\text {aver }}\right)_{x, y} / \varepsilon_{0}\left(E_{\text {aver }}\right)_{x, y}, \\
\left(\mu_{r}\right)_{e f f}=\left(B_{\text {aver }}\right)_{z} / \mu_{0}\left(H_{\text {aver }}\right)_{z} .
\end{gathered}
$$

Since $E_{x, y}$ and $D_{x, y}$ have nearly the same distributions in HRSCs and RCSCs (for metal split tubes and metal cylinders have the same shielding effect for $E_{x, y}$, when $\left.\lambda \gg \Delta\right),\left(\varepsilon_{r}\right)_{\text {eff }}$ in the two crystals are the same, given by the well-known Maxwell-Garnett (exact in long-wavelength and small filling ratio limit) formula: $\left(\varepsilon_{r}\right)_{\mathrm{eff}}=\left(1+f_{s}\right) /\left(1-f_{s}\right)$, where $f_{s}$ $=\pi r^{2} / a^{2}$. However, the distribution of $B_{z}$ is different in the two configurations because $B_{z}$ should be zero in the perfect metal and $H_{z}$ is uniform in the 2D plane due to its continuity. So $\left(\mu_{r}\right)_{\mathrm{eff}} \approx 1$ for HRSCs with thin shells and $\left(\mu_{r}\right)_{\mathrm{eff}} \approx 1-f_{s}$ for RCSCs. As a result, HRSCs have
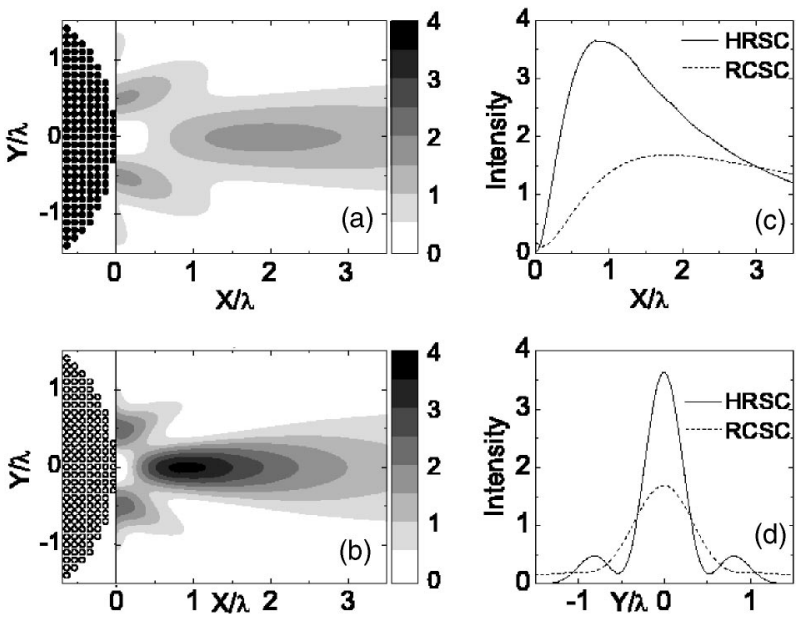

FIG. 4. Focusing a plane wave at the reduced frequency of 0.1 (i.e., $\lambda=10 a$ ) by an acoustic lens based on the HRSC and RCSC: (a) the 2D intensity pattern for the RCSC lens, (b) the 2D intensity pattern for the HRSC lens, (c) the variation of the intensity along the $x$ axis at $y=0$, and (d) the variation of the intensity along the $y$ axis at $x=0.87 \lambda(1.78 \lambda)$ for the HRSC (RCSC) lens. The SCs are same as those in Fig. 1.

$$
n=Z=\sqrt{1+f_{s}} / \sqrt{1-f_{s}},
$$

and RCSCs have

$$
\begin{gathered}
n=\sqrt{1+f_{s}}, \\
Z=\sqrt{1+f_{s}} /\left(1-f_{s}\right) .
\end{gathered}
$$

Note that for RCSCs, $n$ can be increased by increasing $f_{s}$ [2], but at the expense of a rapidly increasing $Z$. It is also clear that the HRSC always has a bigger $n$ and smaller $Z$ than the RCSC because it has bigger $\left(\mu_{r}\right)_{\mathrm{eff}}$ in the EM counterpart. In the above examples, with $f_{s}=0.5,\left(\varepsilon_{r}\right)_{\text {eff }}$ of 3.30 (3.30) and $\left(\mu_{r}\right)_{\text {eff }}$ of $0.86(0.54)$ can be deduced for the HRSC (RCSC) by Eq. (9) and (10) and the FDTD-calculated $n$ and $Z$. The values agree well with above analytic results. In addition, we perform FDTD calculations and obtain the $n$ and $Z$ at low frequencies for both HRSCs and RCSCs with different $f_{s}$ [13]. The results are plotted in Fig. 3(b) compared with the values obtained by Eqs. (13) and (14). We can see that the numerical results are reasonably predicted by the approximate equations.

By now we have shown that our HRSCs have higher $n$ and lower $Z$ than RCSCs. In Fig. 4, we demonstrate the focusing of a plane wave with reduced frequency of $f^{\prime}=0.1$ and intensity of 1 unit by a convex lens based on the crystals in Fig. 1. Absorbing boundary conditions are adopted for all borders in the FDTD scheme. The spatial distributions of the transmitted intensity are shown in Figs. 4(a) and 4(b) for the RCSC and HRSC lenses, respectively. The focusing of the transmitted waves is evident, especially in Fig. 4(b) with our HRSC system. Such a focusing effect is not found for the flat lenses. To quantitatively clarify the focusing effect, the intensity along the $x$ and $y$ axes across the focusing point 
(maximum intensity point) is shown in Figs. 4(c) and 4(d). The focusing length is found to be $0.87 \lambda(1.78 \lambda)$, the normalized intensity at focusing point is 3.63 (1.69), and the transverse full width at half maximum of image is $0.5 \lambda(0.8 \lambda)$ for the HRSC (RCSC) lens. So our HRSC lens will have better focusing effect and better resolution for acoustic imaging.

In the above discussion, we ignored dissipation. Since resonance is used, the viscous loss of air will be important especially for HRs with very narrow apertures [6], so it is necessary to check the absorption of the HRSC. We set our HRSC with real parameters of $a=3.4 \mathrm{~cm}, r=1.36 \mathrm{~cm}$, and $d=\Delta=0.34 \mathrm{~cm}$. Figure 5 shows the absorption of acoustic intensity $A$ for a six-layer HRSC (along the $\Gamma-X$ direction) slab compared with a slab of air with the same thickness of $20.4 \mathrm{~cm}$ [14]. We can see that the absorption of our HRSC is somewhat enhanced by the Helmholtz resonance. However, the absorption is still very low $(A=0.17 \%$ at the absorption peak of $1.3 \mathrm{kHz}$ ) and thus the results in Fig. 4 remain valid even with the absorption considered.

In summary, we have presented a type of sonic crystal based on Helmholtz resonance and show that it can have a high relative acoustic refractive index and a small acoustic impedance mismatch with air, enabling the construction of thin convergent acoustic lens. The numerical results are

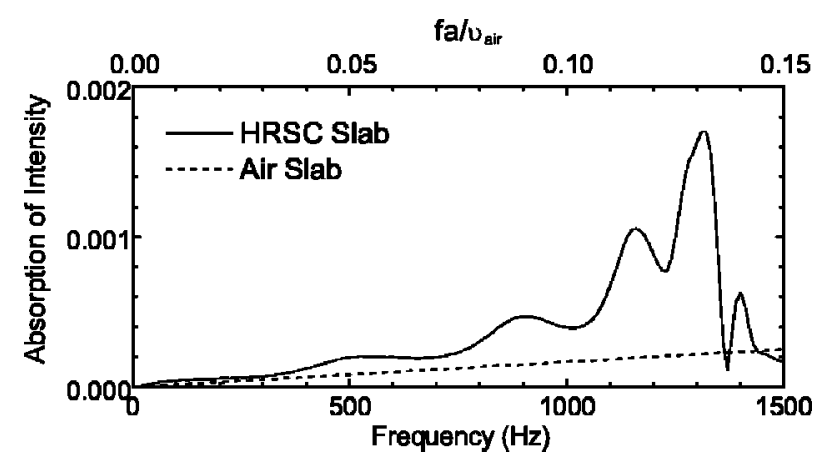

FIG. 5. Absorption spectrum for a six-layer HRSC slab compared with a slab of air with the same thickness. The HRSC has $a=3.4 \mathrm{~cm}, r=1.36 \mathrm{~cm}, d=\Delta=0.34 \mathrm{~cm}$, and the surface normal along the $\Gamma-X$ direction.

based on FDTD calculations and the underlying physics is explained by mapping our results to a corresponding EM model.

This work was supported by RGC Hong Kong through Grant No. CA02/03.SC01 and the Chinese National Key Basic Research Special Fund.
[1] M. M. Sigalas and E. N. Economou, J. Sound Vib. 158, 377 (1992); M. S. Kushwaha et al., Phys. Rev. Lett. 71, 2022 (1993); M. S. Kushwaha, Int. J. Mod. Phys. B 10, 977 (1996).

[2] F. Cervera et al., Phys. Rev. Lett. 88, 023902 (2002); B. C. Gupta and Z. Ye, Phys. Rev. E 67, 036603 (2003); A. A. Krokhin, J. Arriaga, and L. N. Gumen, Phys. Rev. Lett. 91, 264302 (2003).

[3] Z. Liu et al., Science 289, 1734 (2000).

[4] C. Goffaux et al., Phys. Rev. Lett. 88, 225502 (2002).

[5] D. Garcia-Pablos et al., Phys. Rev. Lett. 84, 4349 (2000); Y. Tanaka, Y. Tomoyasu, and S. I. Tamura, Phys. Rev. B 62, 7387 (2000).

[6] L. E. Kinsler et al., Fundamentals of Acoustics (Wiley, New York, 2000).

[7] The HR consists of a rigid-wall cavity of volume $V$ with a neck of area $S$ and length $L$. The air in the neck moves as a mass unit and the acoustic pressure within the cavity provides the stiffness. Such a system supports the Helmholtz resonance at $\omega_{H R} \approx v_{\text {air }} \sqrt{S / L V}$. For our 2D case, $V, S, L$ relate to the area
$A_{s}$ in the tube, the aperture width $\Delta$, and wall thickness $d$, respectively.

[8] Due to the viscous losses of air in the neck at resonance, the HRs are frequently deployed as band acoustic absorbers. But the absorption can be small in the moderate HRs with wide apertures.

[9] C. T. Chan, Q. L. Yu, and K. M. Ho, Phys. Rev. B 51, 16635 (1995); A. Taflove, Computational Electrodynamics: The Finite-Difference Time-Domain Method (Artech House, Boston, 1995).

[10] J. D. Jackson, Classical Electrodynamics, 3rd ed. (Wiley, New York, 1998).

[11] D. R. Smith et al., Phys. Rev. B 65, 195104 (2002).

[12] J. B. Pendry et al., IEEE Trans. Microwave Theory Tech. 47, 2075 (1999).

[13] To produce the same accuracies for different $f_{s}$, the grid length $d x=d y=r / 16$ in all our FDTD calculations.

[14] We use $\rho_{\text {air }}\left(1+4.5 \times 10^{-5} i\right)$ as the mass density of air to simulate the experimental absorption of sound in air. 